\title{
ZÜHLKE, Bärbel, Christine de Pizan in Text und Bild. Zur Selbstdarstellung einer frühhumanistischen Intellektuellen
}

Joseph Morsel

\section{OpenEdition}

Édition électronique

URL : http://journals.openedition.org/ifha/1907

DOI : 10.4000/ifha. 1907

ISSN : 2198-8943

\section{Éditeur}

IFRA - Institut franco-allemand (sciences historiques et sociales)

Référence électronique

Joseph Morsel, « ZÜHLKE, Bärbel, Christine de Pizan in Text und Bild. Zur Selbstdarstellung einer frühhumanistischen Intellektuellen », Revue de l'IFHA [En ligne], Date de recension, mis en ligne le 01 janvier 1995, consulté le 22 septembre 2020. URL : http://journals.openedition.org/ifha/1907 ; DOI : https://doi.org/10.4000/ifha. 1907

Ce document a été généré automatiquement le 22 septembre 2020.

(C)IFHA 


\title{
ZÜHLKE, Bärbel, Christine de Pizan in Text und Bild. Zur Selbstdarstellung einer frühhumanistischen Intellektuellen
}

\author{
Joseph Morsel
}

Si peu d'ouvrages paraissent en France sur l'Allemagne médiévale, il n'en va pas de même pour ce qui est d'ouvrages allemands traitant de thèmes français. La Bourgogne est vraisemblablement la région de France qui intéresse le plus les médiévistes allemands. Géographiquement et historiquement positionnée entre France et Empire, elle bénéficie en outre de l'éclat de la cour et de l'organisation poussée de la principauté de ses derniers ducs Valois, ce qui se traduit aujourd'hui par l'existence d'une riche documentation. C'est sur un type apparemment bien connu de celle-ci, la chronistique bourguignonne, que M.Z. bâtit son ouvrage consacré à la représentation de la politique bourguignonne entre France et Empire. Il ne s'agit pas tant pour lui d'utiliser les diverses chroniques comme autant de sources d'information sur cette politique que d'étudier l'image de la maison de Bourgogne que les chroniqueurs en question entendent livrer à la postérité, en particulier sur le plan des relations avec les deux grands " voisins ». Bref, il s'agit de comparer ce qui nous en est dit dans ces chroniques avec nos connaissances et d'interpréter les écarts observés, issus soit du mode de pensée du chroniqueur, soit d'une mauvaise information, soit d'une volonté de propagande. L'entreprise est conduite en une succession de chapitres consacrés chacun à l'un des chroniqueurs (Monstrelet, Le Fèvre de Saint-Rémy, Wavrin, de Fénin, d'Escouchy, du Clercq, de Haynin, Chastellain, Molinet, de la Marche) et tous bâtis sur le même modèle: l'auteur et l'oeuvre, la Bourgogne et/ou ses ducs face à la France dans l'oeuvre en question, indications sur l'Empire dans l'oeuvre. Un dernier chapitre tente de synthétiser le tout, fondamentalement sur le même modèle: les auteurs et leur oeuvre (vue d'ensemble sur la chronistique bourguignonne et son homogénéité formelle, personnelle, idéelle et temporelle); la Bourgogne et la France (thème principal, donnant aux ducs le beau rôle sans pour autant remettre en cause 
l'attachement au royaume de France); la Bourgogne et l'Empire (thème en définitive rarement évoqué - sauf sous l'angle religieux - et en partie brouillé par l'usage d'une terminologie floue). L'analyse reste toutefois trop peu poussée pour que l'on puisse voir dans cet ouvrage une avancée majeure dans cette connaissance des représentations historiques qui est si importante pour l'histoire sociale.

2 C'est à un autre fantasme historique qu'est consacré l'ouvrage richement documenté de H.M.: la « croisade bourguignonne ", projet toujours réaffirmé par Philippe le Bon (notamment lors du fameux « Voeu du faisan ») mais jamais réalisé. Sont envisagés sous cet angle, de manière chronologique, la création de l'Ordre de la Toison d'Or, les relations avec le Portugal et l'Aragon (dont la duchesse Isabelle constitue un pivot essentiel), la diplomatie bourguignonne vis-à-vis de la France et de l'Empire, le Voeu du faisan, l'échec final du projet (le seul résultat concret étant la libération de Ceuta en 1464) sous la pression de Louis XI et d'une partie de la noblesse bourguignonne. Autour de ce projet se mettent en place les germes de configurations d'alliances qui ont joué à plein au XVIe s. (Habsbourg-Espagne-Papauté contre France-Ottomans) et montrent ainsi combien les fantasmes historiques peuvent avoir été eux-mêmes créateurs de réalité historique.

3 Le chancelier Nicolas Rolin semble avoir joué un rôle déterminant dans la genèse des projets de croisade de Philippe le Bon. Sur le chancelier, on a déjà présenté dans le Bulletin précédent l'ouvrage de Hermann Kamp, consacré à sa pratique mémorielle, mais un nouvel ouvrage, dû à H.-F.P., vient de paraître, présenté comme « la première monographie sur Nicolas Rolin » et qui entend rassembler et recomposer les multiples facettes du chancelier que de nombreux travaux partiels (notamment depuis le XIXe s.) ont déjà mises à jour. Il s'agit donc essentiellement d'un travail historiographique, qui s'ouvre par un rapide panorama du contexte historique (Bourgogne-France-Angleterre) du chancelier. H.-F.P. compile ensuite les travaux historiques concernant Rolin depuis le XVe s. en suivant un plan thématique: origines, études, entrée à la cour, nomination, activités comme chancelier, vie privée, mécénat. Une courte conclusion résume les grands traits de la biographie de Rolin, considéré comme le prototype du conseiller bourgeois des princes du XVe s. Diverses annexes (pièces justificatives, bilan des informations tirées des dictionnaires et encyclopédies, bibliographie) complètent ce curieux ouvrage (une sorte de Que sais-je? sur Nicolas Rolin...).

4 Christine de Pizan jouit quant à elle d'un incontestable succès outre-Rhin, notamment parmi les historiennes. En tant que veuve chargée de famille, contrainte de gagner sa vie et celle de ses enfants et s'étant de ce fait lancée dans la production écrite, devenant ainsi la première femme-écrivain professionnelle de France, elle a inspiré à B.Z. un ouvrage fort bien documenté et qui s'attache à deux thèmes: d'une part l'autoreprésentation (préférée à " autobiographie ») d'une femme (à mi-chemin entre la Christine réelle et une sorte d'idéal-type féminin), présentée de son enfance à son veuvage (conçu comme une "mutacion " de l'identité sociale, à savoir une masculinisation) en passant par son mariage; d'autre part l'étude du rapport entre texte et image, dans la mesure où Christine a semble-t-il attaché tout autant d'importance à l'illustration qu'au texte de ses ouvrages et où de nombreuses miniatures réalisées sous son égide représentent un type spécifique de femme. Les images ne sont toutefois pas conçues comme des illustrations du texte, mais comme un medium complémentaire au texte, précisant notamment l'image de cette femme par le recours au type de la " virago » (figures de Minerve, Sémiramis, la Sybille de Cumes et 
sainte Catherine - c'est-à-dire des femmes à forte personnalité, considérée comme une "personnalité masculine »).

5 Parmi tous les ouvrages de Christine de Pizan, il en est un qui a depuis longtemps retenu l'attention de chercheuses, qui y voyaient l'un des premiers textes féministes: la Cité des Dames. C'est sur cette oeuvre que revient M.K., renvoyant dos-à-dos les adeptes et les contempteurs de l'interprétation féministe, qui laissent de côté un aspect essentiel, le texte, lu jusqu'alors de manière "positiviste ». Les principes qui ont présidé au choix de références et à leur recomposition dans le texte - bref le processus de " création littéraire »- n'ont jusqu'alors pas été étudiés dans le détail, entravant ainsi une bonne compréhension de l'ensemble. M.K. met à jour les procédés allégoriques de Christine et fait ainsi apparaître les attendus de l'ouvrage: il s'agit moins d'élaborer un modèle idéal (ou utopique) de société où les femmes occuperaient une place meilleure que celle que leur réservent les hommes, que de leur faire jouer - et faire en sorte qu'on leur laisse jouer - le rôle qui leur revient dans la restauration de l'ordre social divin et dans la préparation de la communauté au Salut. Bref, il s'agit d'une utopie "conservatrice », bien éloignée du progressisme qu'on a bien volontiers prêté à Christine de Pizan.

6 Le rôle de la « fille aînée de l'Eglise » dans le maintien de l'ordre chrétien a quant à lui rassemblé médiévistes français et allemands lors d'un colloque sur «L'Eglise de France et la Papauté (Xe-XIIIe siècle) ", dont un compte-rendu a été publié dans la revue Francia 18/1 (1991), p.161-164. Les actes du colloque sont parus il y a peu, dans la langue des auteurs, inaugurant une nouvelle collection co-dirigée par l'Institut Historique Allemand à Paris et l'Ecole des Chartes. Le but du colloque était de montrer, sur la base de divers exemples des rapports entre Eglise de France et papauté (situation au Xe s., légats pontificaux, forme des actes épiscopaux, rapports pape/évêque sur la base d'une étude de cas, rapports entre papauté et monastères d'après les exemples de Luxeuil, Corbie, Saint-Denis, Sainte-Geneviève et Saint-Victor de Paris, cas de régions précises - Bretagne, Bourgogne, Franche-Comté, Lotharingie et Catalogne), combien un instrument de travail tel que les Gallia Pontificia (inventaire raisonné des sources de l'histoire des rapports entre l'Eglise de France et la papauté, dont existent déjà de nombreux travaux préparatoires) est nécessaire pour mener à bien des recherches dans ce domaine. On ne peut toutefois que souhaiter que l'édifice des Gallia Pontificia (dont ces actes constituent une pierre angulaire), une fois achevé, restera bien l'utile instrument de travail promis et ne contribuera pas à faire accroire l'existence objective et réelle de rapports entre l'Eglise de France et la papauté par le rassemblement de sources autour de ces hypostases du discours historique, accréditant et conférant un " effet de réel " à ce qui relève en fait de procédures modernes de découpage et de regroupement de sphères initialement distinctes et dont la distinction elle-même fait sens.

7 Joseph MORSEL 